\title{
Intervista a Francesca Duranti
}

\author{
Stefania Lucamante
}

Nel novembre 1993, tre mesi prima della pubblicazione del suo ultimo libro, intitolato Progetto Burlamacchi, Francesca Duranti è venuta a Washington per una serie di conferenze. La visita negli Stati Uniti si doveva, in parte, alla partecipazione della scrittrice alla International Book Fair di Miami, in qualità di prima rappresentante ufficiale italiana presente alla mostra. Nella breve tappa nella capitale che ha preceduto la visita in Florida, la Duranti ha tenuto due conferenze: la prima, svoltasi all'Università del Maryland, proponeva un interessante confronto fra i romanzi di Michel Tournier e quelli di Vincenzo Cerami, o meglio, della rappresentazione dell'uomo borghese che da essi emerge. Nella seconda conferenza, patrocinata dall'Istituto Italiano di Cultura, la scrittrice ha invece parlato a lungo del processo creativo legato alla nascita di un romanzo. Immediatamente prima della conferenza all'Istituto, la Duranti ha concesso la seguente intervista in una sala dell'hotel Ritz-Carlton.

Si parla spesso, e non solo a proposito della nostra letteratura, di una crisi del romanzo in quanto genere letterario. Roberto Cotroneo, nel recensire il nuovo Dizionario Einaudi della Letteratura Italiana del Novecento, curato da Alberto Asor Rosa, decreta la condanna finale per la nostra letteratura, considerandola ormai "finita" (L'Espresso, 20 dicembre 1992). In che misura Lei condivide questa opinione un po' provocatoria di Cotroneo?

Duranti: Innanzi tutto devo dire che io non mi preoccupo delle opinioni di Cotroneo, come non mi preoccupo delle opinioni di qualcuno che è sempre uguale a se stesso. Mi sembra che una persona che ha pensato una volta per tutte non è mai interessante. A me interessano le persone che ogni giomo si alzano e si prendono la briga di pensare, e quindi è possibile che ogni giorno siano diverse dal giomo precedente o che, comunque, abbiano un atteggiamento diverso, che si ricredano, che cambino idea. Chi è uno stroncatore di professione non mi interessa. Diciamo che, comunque, l'idea che ci possa essere una fine del romanzo, in generale, è sbagliata di sicuro. Parlare di una grande crisi del romanzo italiano è anche sbagliato in quanto, se mai, esso soffre di una lentezza di decollo. Non è che ci sia stato e poi sia calato: il romanzo italiano è sempre stato una cosa marginale. Il vero romanzo, e mi riferisco a Il fu Mattia Pascal, o a La coscienza di Zeno sono sempre fenomeni, anche geograficamente, marginali in Italia; anche $L$ 'isola di Arturo è marginale in quanto la donna, a quel tempo, non era poi così centrale nel 
panorama della cultura italiana. Quindi se volessimo definire il romanzo, e non è facile, come un disegno di tenuta estetica, come tracciato, che però sia formato di tessere che contengano eventi, personaggi, storie, si è verificato realmente di rado in Italia. Noi abbiamo molto di più la tendenza alla "bella pagina", molto più la tendenza allo sfogo lirico: noi stiamo costruendo ancora adesso la lingua italiana, e la costruiamo già vecchia senza il coraggio di metterla sotto e dire: "Beh, finalmente la nostra lingua ce l'abbiamo, la possediamo e la usiamo per costruire un romanzo". Non è la fine quindi, ma il mancato "inizio" che dovremmo semmai lamentare. Mancato inizio che però ha delle buone eccezioni.

Esiste un filo nella tradizione del nostro romanzo, fra cui Lei ha citato Il fu Mattia Pascal e L'isola di Arturo, che pensa recuperabile attraverso gli autori contemporanei?

Duranti: Non saprei dirlo. C'è da dire che per tradizione non si può più intendere una tradizione geografica, perché naturalmente la tradizione di ciascuno è l'insieme di quelle suggestioni che lo hanno colpito in un'età in cui non c'era ancora cultura, in cui tutto diventava seconda natura, e quindi queste suggestioni possono essere venute da chi sa dove. Perciò è già difficile dire che cos'è oggi una tradizione; la mia, per esempio, e tutt'altro che in funzione della letteratura italiana. Se dovessi riconoscere in qualche autore contemporaneo una linea mi viene in mente subito quella Consolo-Verga, ma altrimenti non mi viene facilmente e se ci dovessi arrivare sarebbe un po' far-fetched.

Il paradigma letterario si è orientato da una ventina d'anni dalla parte del lettore (o della lettrice). Il testo è "li" e si presenta nella sua pericolosa forma aperta (contenente però certe limitazioni), che il lettore colto può arricchire grazie al proprio background e alla personale competenza letteraria. Lei crede che sia possibile per il testo di essere soggetto ad interpretazioni multiple e, a volte, inequivocabilmente distanti nei loro postulati? Colui che scrive ed il testo stesso mantengono un ruolo determinante e determinato nell'operazione interpretativa?

Duranti: Diciamo che, per quello che faccio, per quello che penso debba essere un romanzo, certamente l'ambiguo, e quindi la risposta aperta, è parte della magia del narrare. Non solo: se, caso mai, il romanzo dovesse avere una funzione (io non credo che ce l'abbia) sarebbe comunque quella di suscitare dei dubbi, non certo quella di dare delle risposte, e quindi di per sé è qualche cosa che poi mette in moto ciascun lettore verso una direzione diversa. Esistono pur sempre delle limitazioni interpretative imposte dalle intenzioni e finalità dell'autore stesso; trascurandole mi sembra che la lettura non possa considerarsi corretta.

Secondo Lalla Romano le donne oggi scrivono meglio degli uomini, "soprattutto perché liberate da una certa tensione nel misurarsi con la scrittura maschile" 
(L'Espresso, 1/8/93). La scrittrice attribuisce alle donne una maggiore libertà espressiva nel trattamento dei temi, e le crede capaci di una scrittura "senza fretta". Lei condivide l'opinione della Romano e, se la risposta è affermativa, ne condivide anche i motivi?

Duranti: No, io non la condivido. Se io dovessi fare un lista degli scrittori italiani che oggi ritengo più interessanti non ci sarebbe nessuna donna. Ritengo interessanti, per ragioni anche opposte, scrittori verso $i$ quali ho delle riserve anche come Giuseppe Pontiggia, a suo modo Aldo Busi, Giampaolo Rugarli (quando non vuole tirare fuori tutto dai cassetti, perché allora ne tira fuori anche troppe di cose), oppure Nico Orengo in certi suoi lavori, e Giorgio Pressburger. Ecco, questi sono scrittori che io ritengo, in misura variabile, interessanti. Se io dovessi dire una scrittrice dalla quale io mi aspetto molto, per ora, non saprei farle un nome.

Pensa che la narrativa femminile di oggi possa contribuire a creare in modo consapevole una valida alternativa intellettuale ad un modello sociologico obsoleto come il patriarcato occidentale, come asserisce il filosofo Gargani? Oppure esistono dei condizionamenti a cui la scrittrice deve ancora soggiacere?

Duranti: Io dubito che esista una scrittura femminile. Io credo veramente che la scrittura femminile tanto più diventa forte quanto più si dimentica di essere femminile. Quando io scrivo io non mi ricordo di essere una donna, non mi ricordo di essere italiana, non mi ricordo di essere alta un metro e sessantacinque, di essere bionda, non mi ricordo di me. Io, di me, mi dimentico. E vero che porto dentro tutto questo, in quello che io scrivo, ma non porto dentro tanto in particolare il fatto di essere una donna. Lo porto dentro così come porto dentro il fatto di essere italiana, di essere bionda, di essere alta un metro e sessantacinque. Porto tutto quello che è la mia esperienza culturale, di vita, i miei condizionamenti biologici e fra tutte queste cose c'è anche, in parte, il fatto di essere una donna. Non penso di portare dentro così in particolare il fatto di essere una donna perché io, insomma, non corro su una pista di biciclette, corro su un'autostrada insieme agli altri. Chi va più forte di me, che vada. Io proverò a riprenderlo. Non voglio un trattamento speciale, non voglio una pista di biciclette. Voglio uno scrittore androgino, pur sapendo che non può essere vero, che al limite non è neanche desiderabile. Si dovrà sempre tenere conto del dato biografico, però questo non vuol dire che ci debba essere sempre quel pensiero fisso: esso nasce da un senso di inferiorità che io rifiuto. Certo, il dato biografico è importante: attivando un verso di un poeta tedesco che dice "Ich hatte schlechte Lehrer, das war eine gute Schule" (io ho avuto dei cattivi maestri, ed è stata una buona scuola) posso dire che ho certamente sviluppato talento, se ne ho, sicuramente grazie agli elementi negativi della mia vita: il fatto che la mia mamma non mi voleva bene, l'incapacità di entrare in un rapporto con la gente, il fatto di essere sostanzialmente silenziosa, dato 
che io non parlo mai quando sono con i miei amici. In realtà io scrivo perché qualcosa la devo pur dire ogni tanto, oppure parlo così quando mi danno un microfono e parlo. Ho sempre l'impressione che a nessuno interessi quello che ho da dire, e quindi in sostanza scrivo; so che quando la cosa è scritta, quello che poi sarà andato in libreria, avrà comprato il mio libro, si sarà messo lì a leggere, evidentemente vuole sentire quello che ho da dire, perché ha fatto tutto questo procedimento e quindi io non sono imbarazzata. Normalmente, non oso parlare. La mia vita, nei suoi lati negativi, mi influenza positivamente.

Il problema del linguaggio del romanzo mi pare sia, prima di tutto, il rapporto che lo scrittore intrattiene con la realtà della sua narrativa. Qual è il suo atteggiamento nei confronti della materia narrata e delle parole che utilizza per narrarla? Un atteggiamento di apparente libertà, di trasparenza o un legame coercitivo, piegando la parola secondo l'uso desiderato?

Duranti: Noi usiamo una lingua, l'italiano, che ha sicuramente ancora delle grandi difficoltà ad essere usata nel romanzo; difficoltà dalle quali bisogna uscire. Quindi la ricerca linguistica che, a mio parere, si impone allo scrittore italiano è quella di sottomettere la lingua (quel "sottometterla" deve essere inteso nel senso di portare la lingua a un grado compatibile con il racconto, toglierle "i vestiti della festa" che normalmente ha quando va sulla carta). L'italiano, quando va nero su bianco, tende ad andare "in falsetto", ad essere impostato, e questo va fatto con estrema delicatezza perché non bisogna esagerare. La scelta linguistica di Gadda, per esempio, andava bene per Gadda. Ma non è una soluzione, come non lo è quella di Pasolini. La scelta dev'essere quella della lingua-strumento. La lingua dev'essere totalmente dominata dallo scrittore, il che significa che uno scrittore non deve mai usare una parola, un'espressione, una virgola che non è stabilmente a casa nei suoi pensieri, nel suo modo di pensare, di esprimersi. Nulla dev'essere mai ricercato. Mentre invece la letteratura italiana è sempre ricercata. C'è sempre per me questo estremo fastidio, quando arrivo alla terza riga di un libro, che incomincio a vedere un tipo di punteggiatura o un uso del lessico che sento non congeniale alla persona che lo usa. Per conto mio è la fine di tutto. Con questo non voglio dire che è sempre sbagliato, ma è sbagliato in seno alla letteratura italiana oggi, perché la porta da sfondare è un'altra, quella della naturalezza, prendendo esempi dalla letteratura anglosassone. Il romanzo nasce lì e c'è poco da dire.

\section{Quali sono gli scrittori stranieri che Lei preferisce?}

Duranti: Lo scrittore che io preferisco è Henry James, dal quale io ho preso moltissimo e che non appare nei miei libri, per quanto qualche volta è stato anche detto. Per esempio ho preso il senso della figura, del "disegno" nel tappeto, di questa cosa che non si vede, però, in realtà tutto questo forma un disegno, e mi rendo conto che sta lì il senso della letteratura. Nutro grandissima ammirazione 
per la capacità che ha Jane Austen di fare piccole rivoluzioni, che credo siano le più forti, le più durature. Scrivere con un pennino intinto apparentemente di rosa un personaggio come la madre delle ragazze Bennet di Orgoglio e pregiudizio, una madre che non è Medea, o Clitennestra, insomma una madre "orribile", e che tuttavia riesce ad essere così stupida, così volgare, così negativa per le figlie, è una cosa incredibile. Eppure non si percepisce con una gran facilità: l'Ulysses sembra molto più rivoluzionario, ma secondo me la capacità di creare un personaggio simile (la madre delle Bennet) è infinitamente più rivoluzionario. Ammiro Stendhal, infine, per lo straordinario controllo della scrittura che sfugge, infatti, all'esuberanza retorica da cui sono afflitti in generale sia gli scrittori italiani che quelli francesi.

Dopo un inizio in cui lo sfondo autobiografico mantiene un certo rilievo (vedi La Bambina, Piazza, mia bella piazza) ecco il grande salto al mistery novel, al romanzo gotico, nel 1984 con il premiatissimo La casa sul lago della luna. Come è avvenuto questo passaggio dal familiare, dal romanzo tinto di rosa (cosi com'è stato definito) al romanzo direi colto, con riferimenti alla Mitteleuropa, all' uso del topos del libro scomparso?

Duranti: Mi sembra che sia avvenuta con la massima naturalezza. Io avevo già cominciato a scrivere da ragazzina (allora però scrivevo cosette), provvista di quel bagaglio di "ossessioni" - secondo me necessario - che serve per poter diventare uno scrittore. Quello che mi mancava era la disciplina per portare a termine il lavoro e quindi, in realta, nulla era pubblicabile. Poi c'è stato un giorno in cui ho capito da che punto dovevo cominciare per scrivere un libro e, una volta trovato il punto focale di tutto, cioè il rapporto molto difficile e per niente "rosa" con mia madre, senza neanche bisogno di disciplina, è venuto fuori $L a$ Bambina. Di lì pero, ho imparato in qualche maniera la disciplina. Ho imparato a star seduta, a scrivere quando non se ne ha più voglia, ho imparato non solo come si apre ma anche come si chiude un libro e come si arriva in fondo e ho scritto subito Piazza, mia bella piazza. Questo è stato un libro in qualche maniera d'occasione, poiché il precedente aveva decretato la fine del mio felicissimo matrimonio, non avendo mio marito accettato l'idea di avere una moglie che scriveva. L'ho scritto perche dentro di me sentivo: "Ma come hai messo su questo po' di macello perché volevi scrivere, adesso scrivi, subito!" E allora ho scritto subito un libro, di cui non sono contenta, è un brutto libro. Ritorna di nuovo sul vissuto, perche era quello che premeva; ed infatti è troppo sul personale, troppo poco distaccato, anche se poi l'ho mascherato; non è poi tutto autobiografico: ci sono cose completamente inventate, altre le ho taciute, però ero ancora "sotto pressione". Fatto questo, e presa la mano allo scrivere, alla disciplina, ho cominciato a scrivere romanzi. La prima parte della mia carriera è quella parte che sempre c'è, preparatoria, e poi sono andata sulla mia strada, nella quale potrebbe fare capolino qualcosa di autobiografico, ma sempre molto rimasticato. 
Sempre a proposito di La casa sul lago della luna, come nasce l'idea del germanista, della Mitteleuropa, dell'Austria?

Duranti: Dovendo essere un romanzo in cui c'è questa specie di doppio scambio nel tempo fra Maria Lettner, che diventa vera, e Fabrizio Garrone, che sparisce. Secondo me l'Austria è proprio un luogo dove cose del genere sembra che possano accadere. Eे un paese che sembra una casa tenuta pronta per i proprietari che non ci sono, ma che potrebbero ritornare. Questa è l'impressione che dà a me l'Austria: la passata grandezza non c'è più affatto, ma è come presente nelle strutture, come se gli Asburgo dovessero uscire dalla cripta dei Cappuccini da un momento all' altro per riprendere possesso dell'impero.

In occasione della pubblicazione di Happy Ending, (versione inglese di Lieto fine), la critica americana Bertha Harris ha notato, soprattutto a proposito delle complicate vite sessuali dei personaggi, delle soluzioni simili a quelle della scrittrice inglese Iris Murdoch. Riflettendo sull' incidenza delle commedie shakespeariane sull' impianto narrativo e sulla caratterizzazione dei personaggi nei romanzi della Murdoch, le sembra appropriato suggerire delle influenze shakespeariane anche nel suo romanzo? E Aldo, aggiungerei io, non Le sembra un Prospero toscano?

Duranti: Non ci ho mai pensato, ma è vero. D'altra parte gli archetipi sono lì e li usiamo continuamente. Riflettendoci bene, si potrebbero trovare altre analogie. Il tema che io volevo mettere in risalto è quello dello snobismo. È un tema importante perché oggi che le differenze economiche si possono annullare in breve tempo, rimangono tuttavia a rodere nel fondo le differenze di tipo sociale, che creano degli spostati più di quanto si possa immaginare. Esistono delle persone che, pur di omologarsi a qualcosa o qualcuno che credono superiore, cancellano tutto quello che corrisponde alla propria eredità e alla propria identità, camuffandosi in un ambiente non loro. Secondo me, costoro patiscono il destino di Sisifo perché, nel momento stesso in cui raggiungono un certo scalino, per il solo fatto di averlo raggiunto, non sono contenti e subito guardano al gradino superiore. Quindi, tutto questo scaturisce da una specie di profondo disprezzo di sé e si può riassumere in quella frase che credo sia di Groucho Marx, riportata da Woody Allen: "A me non interessa proprio di far parte di un club che ammetta me fra i suoi membri!". E quindi il disgraziato snob, che non è contento della propria eredità culturale, familiare, va sempre disperatamente in cerca di qualcosa di nuovo. Io ho voluto fare un personaggio roso da questo tarlo, il quale ha "la vita fuori di sé", nel senso che quello che è il centro del suo interesse è fuori dalla propria vita, per definizione, per natura. Quindi Aldo compra questa torre, e da lì osserva col binocolo una vita (quella trascorsa nelle tre ville dagli altri personaggi del romanzo) che non è la sua. Quell'esistenza vissuta dagli aristocratici suoi vicini diventa in qualche maniera la sua, che per lui risulta possibile condur- 
re solo dal di fuori. Il lieto fine è in realtà tristissimo perché, quando Lavinia diventa sua, lui già si distrae, non ne ha più voglia, quella donna lì non gli interessa più. Tutto ciò io l'ho detto con tale, diciamo, misura che in Italia nessuno se n'è accorto: tutti l'hanno preso per un vero lieto fine, senza afferrare la sottile sfumatura ironica di questo "lieto fine".

Il post-moderno, cosi ricco di un rinnovato uso di figure retoriche, di concettismi, ha lasciato al romanziere l' eredità della metafiction che, appropriandosi e dilatando tecniche care al Selfbegetting novel, mette a nudo i meccanismi scrittori con il proposito di depistare il lettore. Nel suo recente lavoro Ultima stesura il personaggio della scrittrice svela il procedimento di riscrittura del già narrato. Lo fa per chiarire la pista interpretativa del suo lettore virtuale, di cui lei anticipa le reazioni, o si compiace piuttosto nell' intento di sviare l'altro lettore, ignaro di quel che è già stato "scritto"?

Duranti: La mia intenzione era, in realtà, un po' diversa, e cioè né di sviare né di condurre ma, piuttosto, di fare una specie di autoritratto involontario, e cioè una specie di proporzione come si faceva a scuola media in matematica - A sta a B come $\mathrm{C}$ sta a D - una proporzione che si chiama, se non sbaglio, continua. In questo caso Francesca sta a Teodora come Teodora sta all'avvocato Righetta o a Eleuteria Papastratos. Cioè, Io-autore sto alla mia autrice immaginaria come la mia autrice immaginaria sta alle cose che sta scrivendo o che ha scritto. In un certo senso, ciò corrisponde a fornire una chiave di lettura (visto che poi tutti stanno al buco della serratura per guardare chi è l'autore che sta dentro), ma che è depistante come potrebbe esserlo in un giallo in cui si danno tutti gli elementi e poi, in realtà, nessuno arriva alla soluzione. Si dà una chiave falsa, in qualche modo, per arrivare, partendo dal più estraneo dei personaggi dentro i racconti, attraverso Teodora, a me. Ed io, nel giro opposto, volevo arrivare al lettore. Ecco, questa era l'idea. Contemporaneamente c'era l'idea di fare (anche lì si trattava di un disegno) una figura nel tappeto che avevo in mente, un mosaico di tessere comiche quasi, per formare un ritratto tragico. Il ritratto della scrittrice non è un ritratto allegro, infatti, però è formato da tutti questi pezzettini che si trovano nei racconti, e che sono, quasi sempre, di genere comico-grottesco, almeno in parte.

In Ultima stesura $i$ due caratteri grafici separano il narrato che è di solito svolto in terza persona maschile, dall'operazione del "narrare" che usa la prima femminile. Che importanza ha nella dinamica del testo quest'uso differenziato del narratore?

Duranti: Dal punto di vista formale tendo sempre a rendere, nei limiti del possibile, più facile la comprensione di quel che io scrivo, perché, secondo me, un'acqua profonda è opportuno che sia chiara. Il torbido, in genere, si fa quando si vuole mascherare un fondale basso. Allora, se uno ha la consapevolezza di avere qualche cosa da raccontare - è vero che la cosa in sé è ambigua, e quindi è 
vero che presenta delle difficoltà inevitabili, ma la ricerca di un sentiero inesplorato presenta in sé delle difficoltà - appesantire il testo con altre difficoltà - o di carattere linguistico o di altro genere, là dove di complessità ce ne sono anche troppe - secondo me è un errore, una stupidaggine. I due caratteri scaturiscono dalla necessità che il lettore non si confonda: che il lettore sappia che, quando c'è il corsivo parla Teodora, e quindi siamo fuori del racconto e siamo nel "raccontare", quando si ritorna al tondo siamo dentro il racconto, allora il protagonista è l'avvocato Righetta, o Eleuteria Papastratos e si ritorna lì. Però anche quello che racconta Teodora di sé è sempre fictional, tanto è vero che lei nell'ottavo racconto scrive che ormai si è raggiunto il quarto grado del "Meta": Teodora che racconta la storia del metronotte aspirante romanziere che redige il racconto di un altro racconto, richiamando l'uso della figura di Sheherazade.

\section{Potrebbe indicare il racconto più significativo?}

Duranti: Forse di tutti i racconti il più importante è "La carezza di Dio", oltre all'ultimo (l'ottavo) per questo grande ritorno di piani. Ma "La carezza di Dio" ha il pregio di avere messo in moto tutto il libro: è l'unico racconto che avevo già scritto, e tutti gli altri li ho scritti apposta per il libro (è falsa l'asserzione che fossero vecchi racconti). Il fatto che io abbia iniziato dicendo "Questo è un vecchio racconto ..." ha diminuito l'interesse per questo libro, perché si supponeva che fossero vecchi racconti. Ho scritto "La carezza di Dio" subito dopo La casa sul lago della luna. Quella nel libro è la vera "ultima stesura" di un racconto richiestomi prima da un editore genovese e, dopo aver subito modifiche e con un diverso titolo, ripubblicato dalla SIP sulla loro rivista. Nel consegnarlo, però, l'ho riguardato e, nel frattempo, ho percepito nel racconto la presenza di elementi di cui io stessa ero inconsapevole nel corso della prima stesura. Ho capito dov'è che andavo a parare, chi era quel personaggio che scriveva, insomma, la viaggiatrice che parlava col signor Bianchini, e perché aveva delle reazioni di tanto fastidio rispetto alla donna che lui raccontava, la Nina. Allora ho sviluppato le cose che mi interessavano di più. Quando era nella cinquina per il Premio Campiello (vinto dalla Duranti nel 1984, n.d.r.), mi chiesero di pubblicare un saggio di scrittura, insieme a quelli degli altri quattro. Inutile dire che, non avendo nient'altro di pronto, 1 'ho riscritto una terza volta, ancora diverso, facendone un'altra stesura. Ho capito poi che poteva essere bella quest'idea dell' "ultima stesura": scrivere un libro in cui ci fosse questa chiarificazione dell'autore rispetto a quello che meditava di scrivere, nel momento in cui lo scrive per l'ultima volta, e questa presa di coscienza ("che brutta espressione!") della propria volontà di mascherarsi dentro un racconto. E quindi ho concepito tutto un romanzo che avesse come centro questo racconto con il finale sospeso, ripreso nel momento in cui lei va a vedere i luoghi del racconto e trova finalmente la soluzione, il motivo per cui quest'uomo era così innamorato. Di lì è partita l'idea di fare tutti questi racconti in maniera analoga: sembra un libro molto semplice, ma in realtà è una cosa 
molto elaborata.

All' inizio del settimo racconto Teodora scrive: "Il settimo racconto è autobiografico, potrebbe anche essere scritto in corsivo". Che cosa vuol dire?

Duranti: Teodora mette questo in mezzo agli altri racconti ma non è fiction, è l'unico racconto veramente su se stessa, per cui potrebbe essere messo in corsivo, ma l'ho lasciato in tondo; anche se il personaggio principale è lei, rimane pur sempre un racconto. $E$ un racconto perché è la storia della sua incapacità di entrare in contatto col suo prossimo, dove c'è amore non ci riesce, perché sciupa tutto, sebbene non si parli di amore, c'è solamente amicizia, e non riesce a mantenere neanche quella.

$* * * * * * * * * *$

Riflettendo sulle risposte offerte dalla Duranti, il suo inserimento in uno specifico filone di scrittura femminile si delinea problematico. Mi sembra invece piu proficuo osservare come i tentativi da lei intrapresi in diverse direzioni del genere romanzesco, la sua capacità di sperimentazione sempre al limite del gioco logicomatematico, oltre alla frequentata tradizione romanzesca straniera di cui lei pone al vertice le opere di Henry James per la loro ricerca formale, e quelle di Marcel Proust per la magistrale capacità di tratteggiare la tipologia della classe agiata, la portino a realizzare un tessuto narrativo decisamente originale. Non risulta pertanto azzardato voler inserire la scrittrice, il cui percorso di lavoro si avvia nella tradizione, ma che pure si spinge oltre di essa con un rinnovato impiego di tecniche e strategie narrative di pesante eredita, come il "point of view" jamesiano, in un orizzonte letterario di più largo respiro di quello in cui, talvolta, sono stati relegati i suoi lavori. Il fascino che esercita l'ambientazione in un paesaggio puro quale quello offerto dalla campagna toscana, la parola "pulita" e senza concessioni a mode effimere che le pagine dei lavori della Duranti offrono nel porgere al lettore l'espressa volontà autoriale di "suscitare dubbi" circa lo stato delle cose, il bisogno di razionalizzare il presente in un sistema quantificabile (e risolvibile) rimangono fra le attrazioni maggiori della sua scrittura.

Georgetown University 